\title{
A!
}

This is an electronic reprint of the original article.

This reprint may differ from the original in pagination and typographic detail.

Nguyen, Minh-Kha; Kuzyk, Anton

\section{Reconfigurable Chiral Plasmonics beyond Single Chiral Centers}

\section{Published in:}

ACS Nano

DOI:

10.1021/acsnano.9b09179

Published: 01/01/2019

Document Version

Peer reviewed version

Please cite the original version:

Nguyen, M-K., \& Kuzyk, A. (2019). Reconfigurable Chiral Plasmonics beyond Single Chiral Centers. ACS Nano, 13(12), 13615-13619. https://doi.org/10.1021/acsnano.9b09179

This material is protected by copyright and other intellectual property rights, and duplication or sale of all or part of any of the repository collections is not permitted, except that material may be duplicated by you for your research use or educational purposes in electronic or print form. You must obtain permission for any other use. Electronic or print copies may not be offered, whether for sale or otherwise to anyone who is not an authorised user. 


\section{Reconfigurable Chiral Plasmonics beyond Single}

\section{Chiral Centers}

Minh-Kha Nguyen ${ }^{\dagger,+}$, Anton Kuzy $k^{\dagger^{* *}}$

$\dagger$ Department of Neuroscience and Biomedical Engineering, Aalto University School of

Science, P.O. Box 12200, FI-00076 Aalto, Finland

$\$$ Faculty of Chemical Engineering, HCMC University of Technology, VNU-HCM, Ho Chi

Minh City, Vietnam

ABSTRACT: Understanding how the geometrical property of chirality is transferred into the physical properties of chiral materials is becoming increasingly important in various research fields, including plasmonic. Advances in DNA nanotechnology, especially DNA origami technique, enabled routine fabrication of complex chiral plasmonics assemblies. However, most of the work has been dealing with plasmonic enantiomers. The concept of multiple chiral centers in stereochemistry provides simple guidelines for generating multiple chiral configurations besides enantiomers. In this issue of ACS Nano, Qiangbin Wang et al. report DNA origami-based assembly and characterization of reconfigurable plasmonic chiral stereoisomers with up to three chiral centers. This Perspective explores the implication of these results for further development of functional chiral plasmonic systems. 
Chirality is a geometrical property and an object is chiral if it cannot be superimposed on its mirror image. Two non-superimposable mirrored configurations are called enantiomers. Chirality is ubiquitous at all scales ${ }^{1}$. The term chiral is derived from the Greek word for hand $\chi \varepsilon i ́ p$ (cheir), and human hands are, perhaps, the most frequently used examples of chiral macroscale objects. Biological macromolecules, e.g., DNA, RNA and proteins, typically have chiral tertiary structures, and their building blocks, i.e., sugars, nucleotides and amino acids are also chiral. Chirality is omnipresent among small organic molecules as a result of the tetravalency of asymmetric carbon. Due to the structure-function relationship chirality plays fundamental role in chemistry and biochemistry. The implications of molecular chirality are broad, and its manifestations span from peculiar to vital. For example, enantiomers can have different odours ${ }^{2}$ and taste. ${ }^{3}$ More critical are therapeutic effects of chiral drugs, where one enantiomer can be useful, whereas the other may be inactive or even harmful. ${ }^{4}$ Understanding relationship between geometrical property of chirality and physical properties of chiral materials is becoming increasingly important to various disciplines besides biochemistry. ${ }^{1}$ One of the such areas is rapidly growing research field of chiral plasmonics that explores optical properties of chiral metal nanostructures. ${ }^{5}$ Fabrication of chiral metal nanostructures typically requires ability to generate genuinely three-dimensional architectures, which remains challenging with the conventional top-down nanofabrication approaches. Molecular selfassembly offers an alternative fabrication approach. ${ }^{6}$ In particular, DNA origami technique ${ }^{7}$ has emerged as a versatile platform for generating complex self-assembled plasmonic nanostructures ${ }^{8,9}$, including chiral architectures. ${ }^{10,11}$ The utility of DNA origami technique for generating chiral plasmonic assemblies with tailored optical responses was first demonstrated in $2012^{12}$ by Liedl and coworkers, who used 24 helix DNA origami bundles as templates to arrange spherical gold metal nanoparticles (AuNPs) into the left-handed (L) and right-handed 


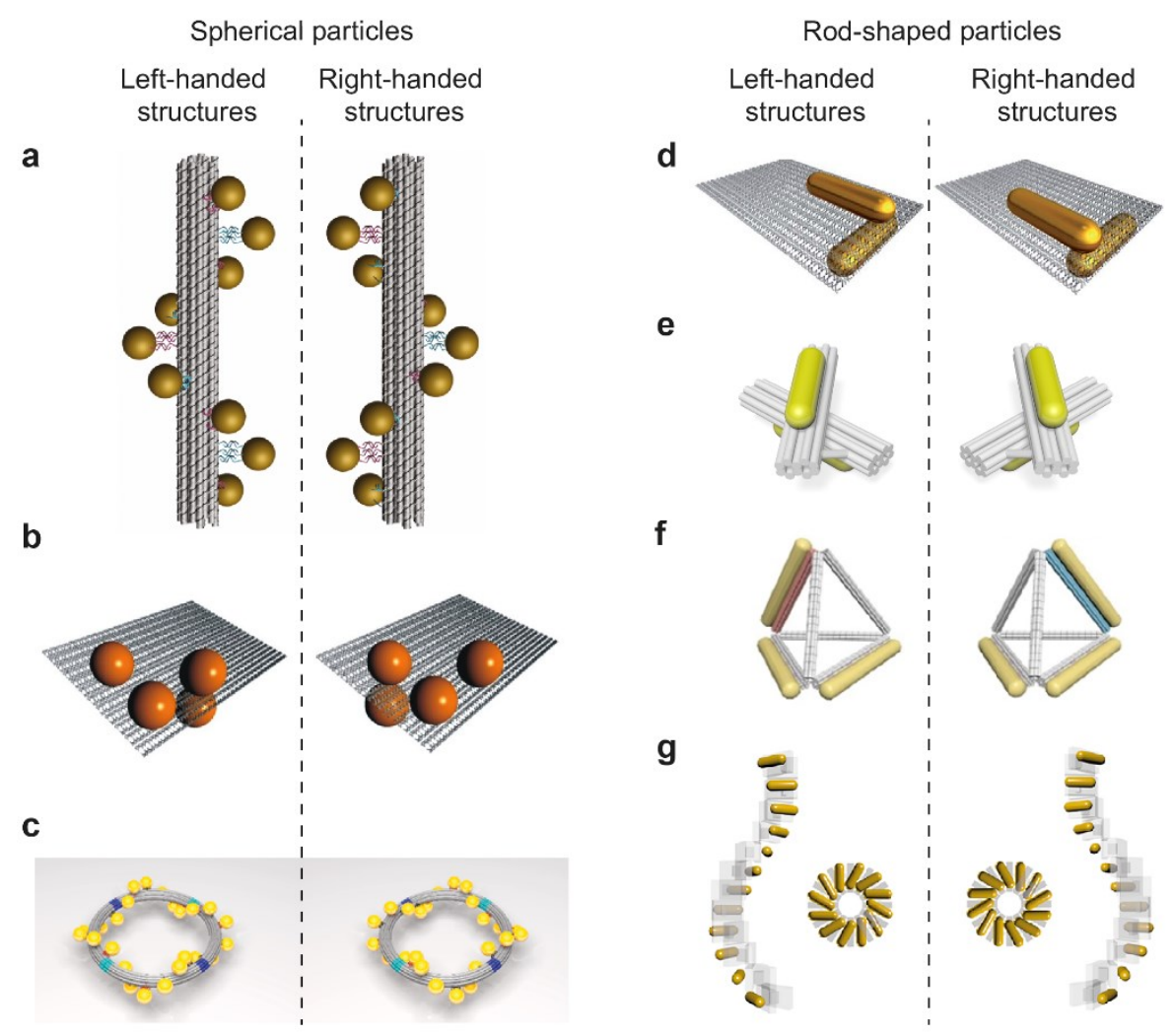

Figure 1. DNA origami-templated chiral plasmonic assemblies of spherical and rod-shaped gold nanoparticles. (a) Left- and right-handed helices of nine $10 \mathrm{~nm}$ spherical AuNPs. Adapted with permission from ref 12. (b) Four spherical AuNPs in right- and left-handed configurations. Four is the minimum number of spherical particles required for achieving three-dimensional chirality. Adapted from ref 13. Copyright 2013 American Chemical Society. (c) Twenty-four AuNPs arranged in helical fashion on DNA origami ring template. Adapted from ref 14. Copyright 2016 American Chemical Society. (d) Chiral plasmonic structures of gold nanorod dimers. Two rods are sufficient for achieving three-dimensional chirality. Adapted from ref 15 with permission from The Royal Society of Chemistry. (e) Chiral plasmonic assemblies with dynamically controlled optical responses enabled by stimuli-responsive DNA origami templates. Adapted from refs 16-18. (f) System in which chirality can be switched by attachment/removal of gold nanorods at specific edges of tetrahedron-shaped DNA origami template. Adapted from ref 19. Copyright 2019 American Chemical Society. (g) DNA origami- 
guided plasmonic helices of nanorods with switchable chirality. Adapted from ref 20. Copyright 2018 American Chemical Society.

(R) helices (see Figure 1a). Collective interactions of AuNPs plasmonic excitations in helical assemblies gave rise to strong optical activity in the visible spectral range. Since then, DNA origami has been widely utilized for fabrication of chiral plasmonic nanostructures. However, most of the works focused primarily on achieving plasmonic optical activity and chiral configuration were typically limited to two enantiomers ${ }^{13-20}$ (see Figure 1). This is mainly due to rather intuitive comparison of spatial configurations and chiroptical responses of enantiomers. Structurally enantiomers are mirror images of each other. Optical responses of chiral materials are usually characterized in terms of circular dichroism (CD), which is defined as the difference in extinctions of the left- and right-handed circular polarized light. The CD responses of enantiomers have the same amplitude but opposite signs.

a

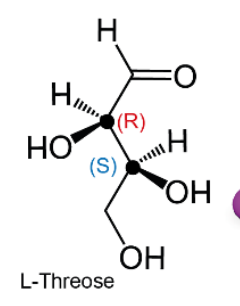

Molecular stereoisomers

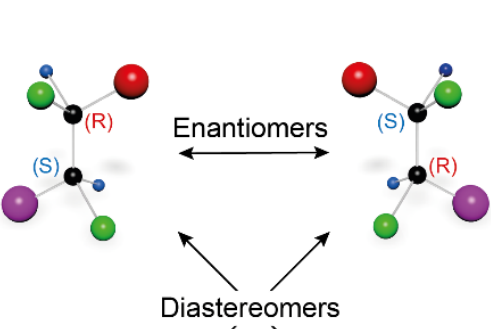<smiles>O=C[C@@H](O)[C@@H](O)CO</smiles>

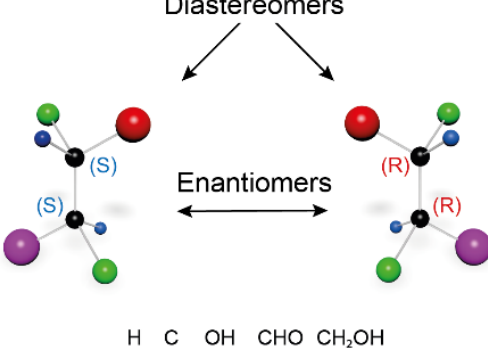

$\mathrm{H} \mathrm{C} \mathrm{OH} \mathrm{CHO} \mathrm{CH}_{2} \mathrm{OH}$

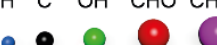

b
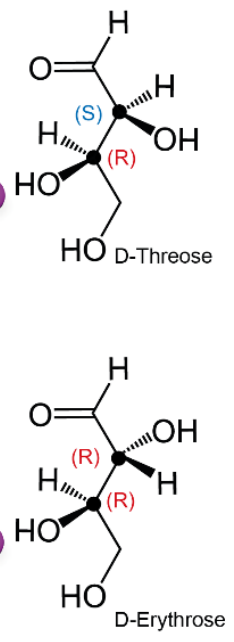
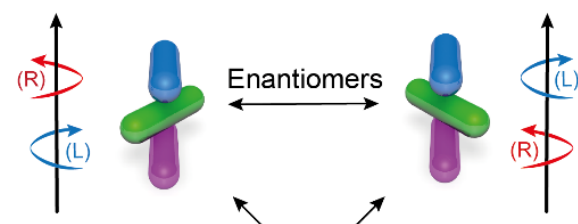

Diastereomers

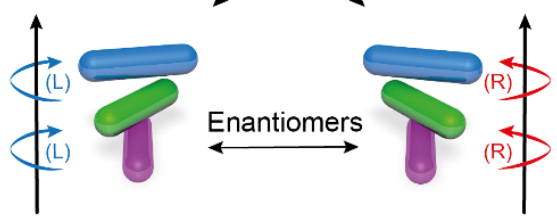

Figure 2. Example of molecular stereoisomers and their plasmonic analogs. (a) Natta projections and schematic illustrations of four stereoisomers of 2,3,4-trihydroxybutanal that has two chiral centers. Stereoisomers that differ in handedness of all chiral centers are enantiomers. (b) Plasmonic stereoisomers of nanorod trimer with two chiral centers. Chiral configuration of two neighboring rods can be either left- (L) or right-handed (R). 
It is well known, at least to chemists ${ }^{21}$, that stereochemistry is not limited to enantiomers (see Figure 2a). Molecules can have multiple chiral centers. Each chiral center can be either in socalled (R) (from rectus, Latin for right) or (S) (from sinister, Latin for left) configuration. The $\mathrm{R} / \mathrm{S}$ configuration is defined according to Cahn-Ingold-Prelog priority rules. A molecule with $n$ chiral centers can have $2^{n}$ stereoisomers. Two stereoisomers that differ in handedness of up to $n-1$ chiral centers are called diastereomers. Diastereomers can have very different chemical and physical properties including different chiroptical responses. Enantiomers are special case of stereoisomers as they differ in handedness at all chiral centers. Chemical and physical properties of enantiomers are identical in achiral environment, with the exception of interaction with circularly polarized light.

Plasmonics has greatly benefited from adapting molecular approaches for design and characterization of complex plasmonic structures. ${ }^{22,23}$ The concept of multiple chiral centers in stereochemistry provides simple rules for generating large number of different chiral plasmonic configurations using simple building blocks (see Figure 2b). However, controlled fabrication of metal nanostructures with multiple chiral centers has remained technically challenging. Plasmonic structures with two chiral centers and their optical responses were first investigated by Hentschel and co-workers. ${ }^{24}$ Two-step electron beam lithography was used for fabrication. Reliable and reproducible fabrication of complex three-dimensional metal nanostructures is not always trivial with electron beam lithography. In this issue of ACS NANO, Qiangbin Wang and co-workers report DNA origami-based assembly of gold nanorods (AuNRs) into plasmonic structures with up to three chiral centers (see Figure 3 ). ${ }^{25}$ DNA origami templates consist of two-layer rectangular plate and ten-helix bundle linked in the center and can host two AuNRs, one attached to the plate and one to the bundle (see Figure 3a). The relative orientation of the bundle and the plate, and hence of the nanorods, can be actively switched between the rightand left-handed chiral configurations using so-called toehold-mediated DNA strand 
displacement reactions. ${ }^{26}$ Moreover, the assemblies are not limited to single origami template and gold nanorods dimers. By careful design of the assembly process Wang et al. succeeded in

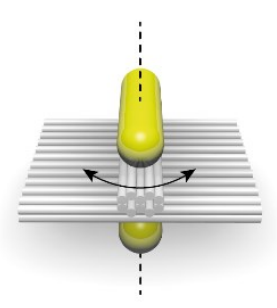

b

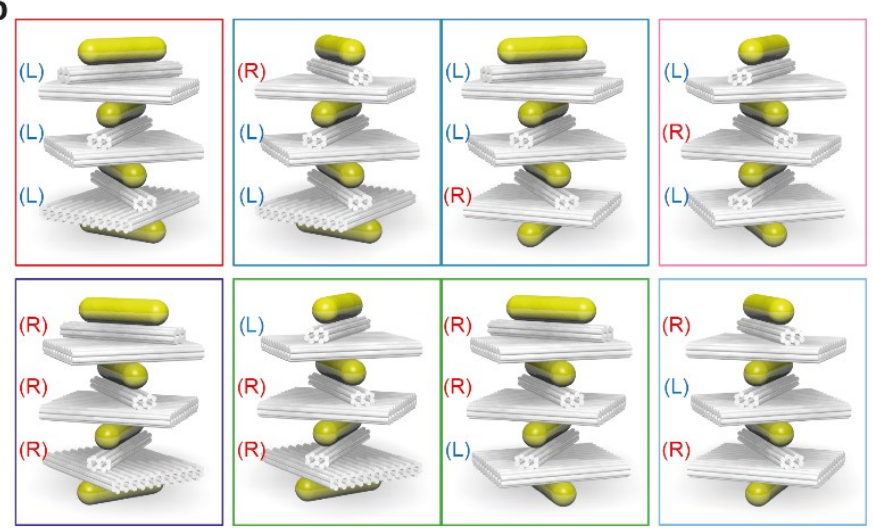

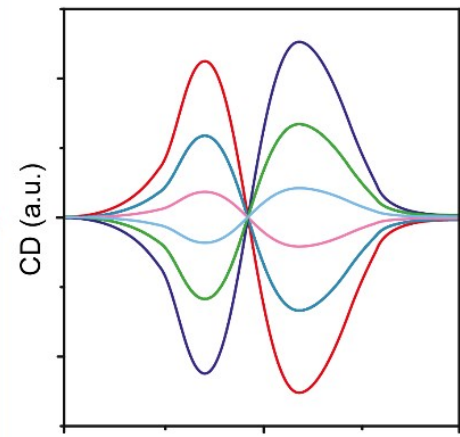

Wavelength $(\mathrm{nm})$

Figure 3. DNA origami-based assembly of chiral plasmonic structures with multiple chiral centers. (a) DNA origami templates are used for assembly of AuNRs. The configuration of origami-AuNRs constructs can be actively switched between right- and left-handed chiral configurations. (b) Four AuNRs and three DNA origami constructs used for assembly of plasmonic structures with three chiral centers, which give rise to eight origami-nanorods stereoisomers and six unique chiral plasmonic spatial configurations. (c) Each plasmonic configuration has very district chiroptical, i.e., $\mathrm{CD}$, response.

arranging up to four AuNRs linked together with DNA origamis. Each pair of neighboring AuNRs can be considered as a chiral center. Crucially, each chiral center can be controllably switched between the L and R configurations. The authors fabricated and characterized AuNRs trimers and tetramers with two and three chiral centers, respectively. Particularly interesting are chiroptical responses of assemblies with three chiral centers, which give rise to eight origami-AuNRs stereoisomers with chiral centers in LLL, RRR, RRL, LLR, LRR, RLL, LRL, RLR configuration (see Figure 3b). Some of those eight stereoisomers are equivalent from the plasmonic point of view, i.e., $R R L=L R R$ and $L L R=R L L$, which brings the number of unique chiral plasmonic configuration down to six. Each of these six configurations has very distinct chiroptical response (see Figure 3c). As expected, LLL and RRR enantiomers, which contain 
only one configuration of chiral centers, showed the strongest chiroptical responses with vertically mirrored CD spectra. Interestingly, responses of stereoisomers that contained both $\mathrm{L}$ and $\mathrm{R}$ configurations depended not only on the number of centers in each configuration but also on their arrangement. For example, both LRL and RLL configurations have one R center and two L centers and exhibit left-handed CD response (see Figure 3c). However, the response of RLL was much stronger. Wang et. al explained this observation in terms of plasmonic coupling between gold nanorods in different centers and concluded that this coupling contributes substantially to the overall CD response.

\section{CONCLUSIONS AND OUTLOOK}

Qiangbin Wang co-workers describe a promising route for assembly of metal nanoparticles into well-defined chiral assemblies with high accuracy and precision. Moreover, the assemblies are endowed with active control over multiple spatial configurations. Both precise assembly and active control are crucial for advancing our understanding of coupled plasmonic systems and realizing their full potential for practical applications. In principle, the assembly approach described by Wang et al. is not limited to chiral configuration and can be adapted for fabrication of various reconfigurable plasmonic architectures.

On more practical side, reconfigurable DNA origami-based chiral plasmonic structures have been recently explored for biosensing applications. ${ }^{27-29}$ Target-probe interactions, transduction mechanisms, and output signals of DNA origami-based sensing probes can be tailored to specific needs due to the high structural and thermodynamic programmability of DNA. In addition, chiral plasmonic assemblies can be used for optical detection in strongly absorbing environments. ${ }^{28}$ Structures with selectively controlled multiple chiral centers might open new routes for development of sensing platform that allow reliable simultaneous detection of multiple targets, which is increasingly important for diagnostic applications. 
The field of DNA-based chiral plasmonic faces several challenges. Reproducible fabrication of plasmonic assemblies requires reliable synthesis of metal colloidal nanoparticles with low degree of polydispersity. Polydispersity often leads to CD responses which are significantly weaker and broader than theoretically predicted. DNA origami-based plasmonics could greatly benefit from adopting the recently developed technique for fabrication of metal nanoparticle with low polydispersity. ${ }^{30}$ Furthermore, generation of chiral assemblies with well-defined number of nanoparticles and DNA origami structures usually requires multiple purification steps which leads to reduced fabrication yields. Surface immobilization in combination with incubation-washing cycles might enable high yield fabrication of DNA origami-based plasmonic superstructures with controlled large number of interacting plasmonic particles. Despite of the challenges, DNA origami technique will continue to play an important role as a fabrication platform for assembly of complex plasmonic structures and will enable exciting scientific and technological developments.

\section{AUTHOR INFORMATION}

\section{Corresponding Author}

*E-Mail: anton.kuzyk@aalto.fi

\section{ORCID}

Minh-Kha Nguyen: 0000-0003-3456-3162

Anton Kuzyk: 0000-0001-8060-6122

\section{Notes}

The authors declare no competing financial interest.

\section{ACKNOWLEDGMENT}


The authors acknowledge financial support by the Academy of Finland (grants 308992 and 324352).

\section{REFERENCES}

1. Morrow, S. M., Bissette, A. J. \& Fletcher, S. P. Transmission of chirality through space and across length scales. Nat. Nanotechnol. 12, 410-419 (2017).

2. Friedman, L. \& Miller, J. G. Odor Incongruity and Chirality. Science 172, 1044-1046 (1971).

3. Gal, J. The Discovery of Stereoselectivity at Biological Receptors: Arnaldo Piutti and the Taste of the Asparagine Enantiomers-History and Analysis on the 125th Anniversary. Chirality 24, 959-976 (2012).

4. Eichelbaum, M. Side effects and toxic reactions of chiral drugs: A clinical perspective. in Toxicology in Transition (eds. Degen, G. H., Seiler, J. P. \& Bentley, P.) 514-521 (Springer, 1995). doi:10.1007/978-3-642-79451-3_44.

5. Hentschel, M., Schäferling, M., Duan, X., Giessen, H. \& Liu, N. Chiral plasmonics. Sci. Adv. 3, e1602735 (2017).

6. Urban, M. J. et al. Chiral Plasmonic Nanostructures Enabled by Bottom-Up Approaches. Annu. Rev. Phys. Chem. 70, 275-299 (2019).

7. Rothemund, P. W. K. Folding DNA to create nanoscale shapes and patterns. Nature 440, 297-302 (2006).

8. Liu, N. \& Liedl, T. DNA-Assembled Advanced Plasmonic Architectures. Chem. Rev. 118, 3032-3053 (2018). 
9. Kuzyk, A., Jungmann, R., Acuna, G. P. \& Liu, N. DNA Origami Route for Nanophotonics. ACS Photonics 5, 1151-1163 (2018).

10. Cecconello, A. \& Simmel, F. C. Controlling Chirality across Length Scales using DNA. Small 15, 1805419 (2019).

11. Zhou, C., Duan, X. \& Liu, N. DNA-Nanotechnology-Enabled Chiral Plasmonics: From Static to Dynamic. Acc. Chem. Res. 50, 2906-2914 (2017).

12. Kuzyk, A. et al. DNA-based self-assembly of chiral plasmonic nanostructures with tailored optical response. Nature 483, 311-314 (2012).

13. Shen, X. et al. Three-Dimensional Plasmonic Chiral Tetramers Assembled by DNA Origami. Nano Lett. 13, 2128-2133 (2013).

14. Urban, M. J. et al. Plasmonic Toroidal Metamolecules Assembled by DNA Origami. $J$. Am. Chem. Soc. 138, 5495-5498 (2016).

15. Shen, X. et al. 3D plasmonic chiral colloids. Nanoscale 6, 2077-2081 (2014).

16. Kuzyk, A. et al. Reconfigurable 3D plasmonic metamolecules. Nat. Mater. 13, 862866 (2014).

17. Kuzyk, A. et al. A light-driven three-dimensional plasmonic nanosystem that translates molecular motion into reversible chiroptical function. Nat. Commun. 7, 1-6 (2016).

18. Kuzyk, A., Urban, M. J., Idili, A., Ricci, F. \& Liu, N. Selective control of reconfigurable chiral plasmonic metamolecules. Sci. Adv. 3, e1602803 (2017).

19. Man, T. et al. Chiral Metamolecules with Active Plasmonic Transition. ACS Nano 13, 4826-4833 (2019). 
20. Lan, X. et al. DNA-Guided Plasmonic Helix with Switchable Chirality. J. Am. Chem. Soc. 140, 11763-11770 (2018).

21. Eliel, E. L., Wilen, S. H. \& Mander, L. N. Stereochemistry of organic compounds. (Wiley \& Sons, 1994).

22. Prodan, E., Radloff, C., Halas, N. J. \& Nordlander, P. A Hybridization Model for the Plasmon Response of Complex Nanostructures. Science 302, 419-422 (2003).

23. Guerrero-Martínez, A., Grzelczak, M. \& Liz-Marzán, L. M. Molecular Thinking for Nanoplasmonic Design. ACS Nano 6, 3655-3662 (2012).

24. Hentschel, M., Schäferling, M., Metzger, B. \& Giessen, H. Plasmonic Diastereomers: Adding up Chiral Centers. Nano Lett. 13, 600-606 (2013).

25. Wang, M. et al. Reconfigurable Plasmonic Diastereomers Assembled by DNA Origami. ACS Nano (2019) doi:10.1021/acsnano.9b06734.

26. Simmel, F. C., Yurke, B. \& Singh, H. R. Principles and Applications of Nucleic Acid Strand Displacement Reactions. Chem. Rev. 119, 6326-6369 (2019).

27. Funck, T., Nicoli, F., Kuzyk, A. \& Liedl, T. Sensing Picomolar Concentrations of RNA Using Switchable Plasmonic Chirality. Angew. Chem. Int. Ed. 57, 13495-13498 (2018).

28. Huang, Y., Nguyen, M.-K., Natarajan, A. K., Nguyen, V. H. \& Kuzyk, A. A DNA Origami-Based Chiral Plasmonic Sensing Device. ACS Appl. Mater. Interfaces 10, 44221$44225(2018)$.

29. Funck, T., Liedl, T. \& Bae, W. Dual Aptamer-Functionalized 3D Plasmonic Metamolecule for Thrombin Sensing. Appl. Sci. 9, 3006 (2019). 
30. González-Rubio, G. et al. Femtosecond laser reshaping yields gold nanorods with ultranarrow surface plasmon resonances. Science 358, 640-644 (2017).

For table of contents use only

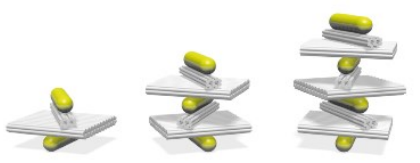

